\title{
Axiomatic Design Aspect of a Braking System Redesign
}

\author{
Kenji Iino $^{1, *}$, Aitor Arruti $^{2}$, and Masayuki $\mathrm{Nakao}^{3}$ \\ ${ }^{1}$ SYDROSE LP, San Jose, California, 95112, USA \\ ${ }^{2}$ Technical University of Denmark, $2800 \mathrm{Kgs}$. Lyngby, Denmark \\ ${ }^{3}$ The University of Tokyo, Hongo. Tokyo 113-0033, Japan
}

\begin{abstract}
A rollator is a commercial product on the market for aiding people with difficulty in walking by themselves. Users are typically strong enough to walk without aid, but will easily feel exhausted within short distances. The product is popular in advanced countries with fairly good walkway conditions with their simple structure and ease of use without any driving power. The product's simple design led to a great number of companies developing their design and putting them quickly in the market. Rushed design, however, sometimes can cause subtle issues with the product. One particular product was found with a drawback in its operability that engaging the hand activated brake can make the following forward movement difficult. This paper discusses the design issue with Axiomatic Design and proposes a remedy that removes the drawback.
\end{abstract}

\section{Introduction}

Our world has a problem of growing population and particularly a higher ratio of elderlies in the coming years. WHO predicts [1] that the ratio of people at age 75 or above, currently at $3.3 \%$, will reach $8.1 \%$ in the year 2055 (Figure 1). Japan, in particular, has a more serious prediction [2] with the same 2055 ratio reaching a stunning $19.7 \%$ from the current $9.1 \%$ (Figure 2).
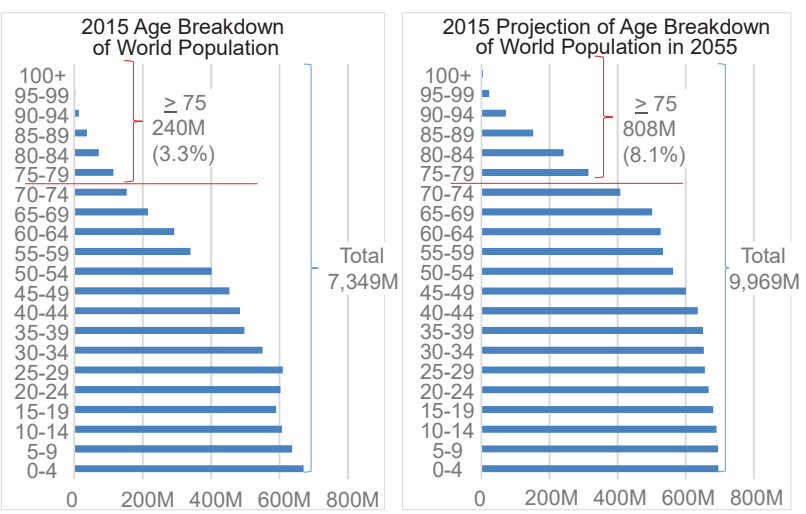

Fig. 1. Age breakdown of world population in 2015 and 2055.

As people age, a number of bodily functions degrade and losing the ability to walk without any support is one of such functions. To help those affected with walking difficulty and disability, tools in the market start from a cane, crutches, a wheel-less walker, a walker with wheels, a rollator, a wheelchair, an electric wheelchair, and a mobility scooter, in the order of increasing level of support and complexity. In the same order, the power of mobilization shifts from human to tool.

\footnotetext{
* Corresponding author: kiino@sydrose.com
}
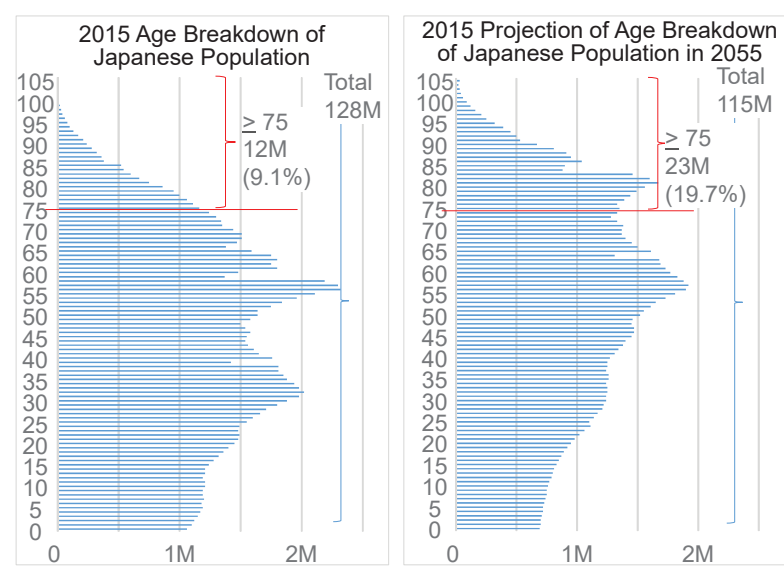

Fig. 2. Age breakdown of Japanese population in 2015 and 2055.

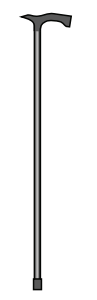

Cane

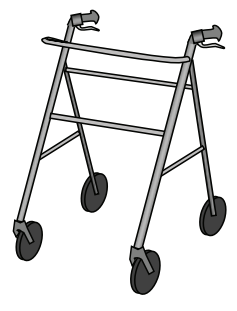

Rollator

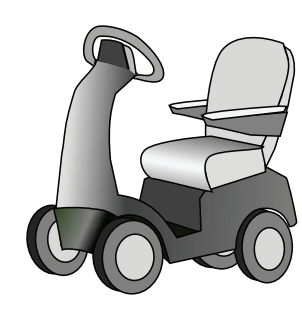

Mobility Scooter
Fig. 3. Walking (mobility) aids with different levels of support.

A specific rollator model caught the attention of the authors and it turned into the topic of our study. Our attention started with Axiomatic Design [3] Analysis of the model's braking system. It worked fine in stopping the rollator, however, the user had difficulty moving forward again from the stopped position. Our analysis 
pinpointed the specific part that was causing the problem. It was a part for stopping the moving rollator that interfered with the functional requirement (FR) of moving the rollator forward.

Our work followed the analysis with redesign of the braking system with a different configuration. The new design removed the interference and freed the forward moving functional requirement from the braking system. In other words, the new design reduced the interfering component in the design matrix negligibly small.

This paper is a report of the results of a 3-month assignment by a visiting student from Denmark.

\section{Original design}

\subsection{High level FR analysis}

The FR for a rollator is to "aid user in walking." Instead of a wheelchair, the user is not fully off the feet, thus, this highest level FR is further broken down to: "partially support body weight," the main FR of "aid forward motion," and the FR in case the user wants to avoid colliding into objects or other people, "stop the motion." Figure 4 is the Design Record Graph [4] for the high level conceptual design of a rollator. The left side of the figure shows the first level decomposition of the highest level FR into the next level FRs.

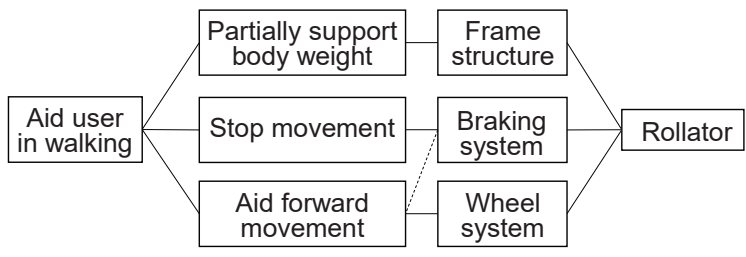

Fig. 4. High level Design Record Graph of rollator.

Our earlier paper [4] pointed out how refining the design into detail can hide interferences among design components because a detail single part tends to have only one functional requirement. Similarly, design interference is hard to identify in early stages of conceptual design because rough sketches of the design concepts without detail still do not have interfering components.

The designer of the rollator probably did not recognize the design interference by the braking system to the FR of "Aid forward movement" which was not intended. It was only after detail design was carried out that a part in the braking system was shaped in a way to interfere with the forward movement. Eq. (1) is the design equation of the high level decomposition. The designer did not intend to have the italic $x$ in the design matrix with the early conceptual design.

$\left\{\begin{array}{c}\text { Partially support body weight } \\ \text { Stop movement } \\ \text { Aid forward movement }\end{array}\right\}=\left[\begin{array}{lll}\mathrm{X} & 0 & 0 \\ 0 & \mathrm{X} & 0 \\ 0 & x & \mathrm{X}\end{array}\right]\left\{\begin{array}{l}\text { Frame structure } \\ \text { Braking system } \\ \text { Wheel system }\end{array}\right\}$

\subsection{Detail of the braking system}

Disassembling the braking system revealed how it transferred the user's grasping force to the braking system to stop the wheel rotation.

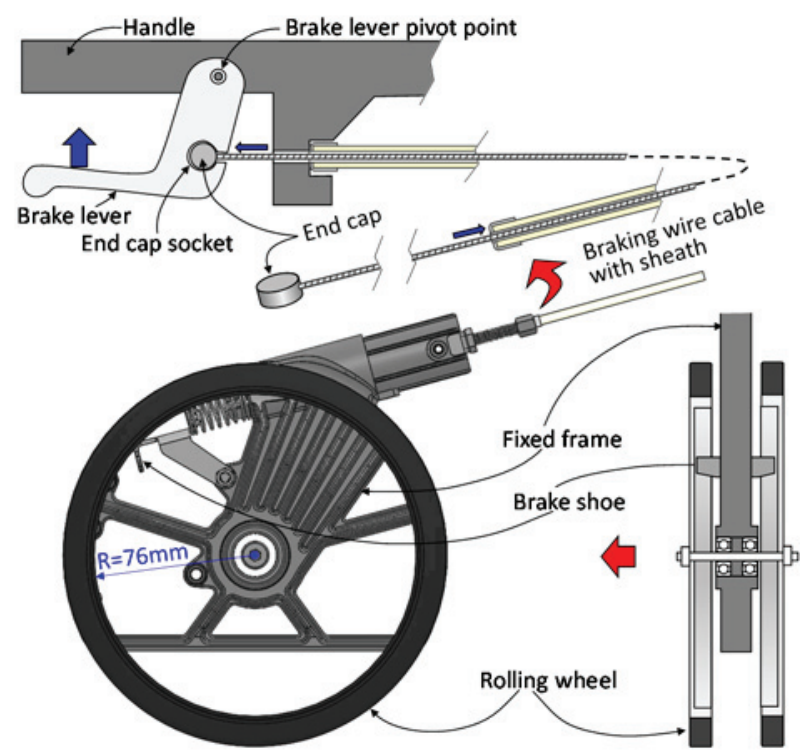

Fig. 5. Original braking system design of our subject rollator.

Figure 5 sketches the wide view of the user engaging the brake. When the user grasps the brake lever with the handle, the brake lever rotates clockwise around its pivot point and the end cap socket pulls the braking wire out from the sheath. At the other end at the wheel, the braking wire is pulled into the sheath. The end cap on the wheel side thus pulls the brake shoe to turn it clockwise around the brake shoe pivot point (Figure 6). The brake shoe then pushes against inside of the wheel rim to apply friction against the wheel to stop its rotation.

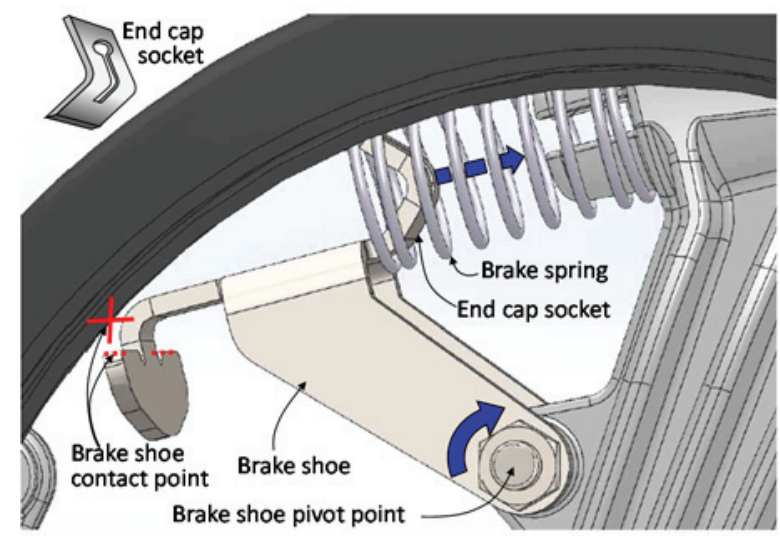

Fig. 6. Engaging the brake to the wheel (Brake shoe at rest).

The upper left corner of Figure 6 shows the slit in the brake shoe that allows placing the end cap in the socket for exerting pulling force on the brake shoe.

The designer placed the brake spring in Figure 6 so that when the user releases the brake lever, it exerts spring force to push the brake shoe back into its rest position, that is, to disengage the shoe from the wheel. 
Figure 7 shows the detail Design Record Graph for this braking system.

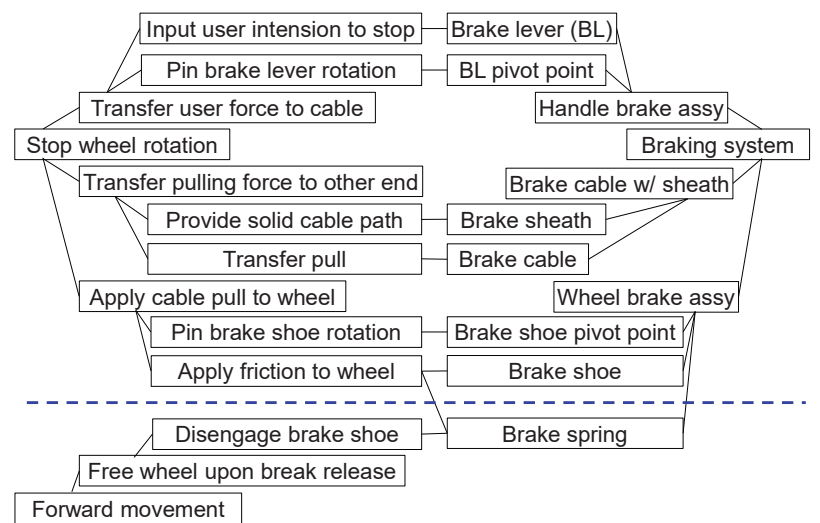

Fig. 7. Design Record Graph of braking system.

Although the brake spring is part of the braking system, its function is to allow forward movement for the rollator from its rest position. Eq. (2) is the design equation for this braking system at a level with more detail than Eq. (1). Note that without the movement FR, the equation shows a completely independent design from an Axiomatic Design aspect.

$\left\{\begin{array}{l}\text { Transfer user force to cable } \\ \text { Transfer pulling force to other end } \\ \text { Apply cable pull to wheel }\end{array}\right\}=\left[\begin{array}{lll}\mathrm{X} & 0 & 0 \\ 0 & \mathrm{x} & 0 \\ 0 & 0 & \mathrm{X}\end{array}\right]\left\{\begin{array}{l}\text { Handle brake assy } \\ \text { Brake cable } \mathrm{w} / \text { sheath } \\ \text { Wheel brake assy }\end{array}\right\}$

Eq. (3) is the design equation at the fine detail level. When applying braking friction to the wheel, the force has to overcome the brake spring force to push the brake shoe away from the wheel rim. The italic $x$ in Eq. (3) shows that the "Friction to wheel" function is affected by the "Brake spring."

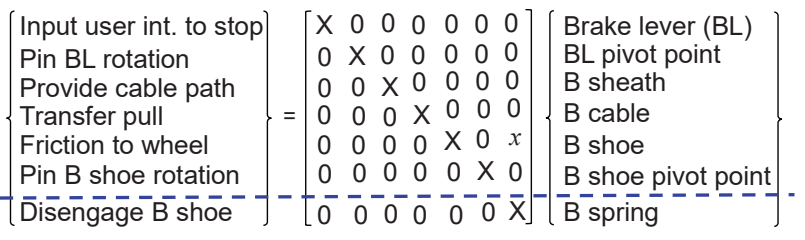

This brake spring is an odd part because physically it belongs to the braking system, however, its function is a sub-function of the function of aiding forward movement. The broken blue lines in Figure 7 and Eq. (3) indicate the functional split of braking and moving forward.

\subsection{Problem with the original design}

Figure 8 shows the brake shoe when the brake is engaged to stop the wheel from rotating. The red curve is the inner surface of the wheel rim where the brake shoe makes contact for braking.

As the figure shows, when the brake cable is pulled to the right, the brake shoe, with wings extending towards the two wheels that sandwich the frame, contacts the inner surfaces of the wheel rims. People, however, tend to exert more than just enough force in braking a moving object. The readers can recall their grasp on bicycle brake levers when they are stopped at an intersection. It is a natural action for a human rider who does not want the bicycle to start moving unintentionally when waiting for the cross traffic to stop.

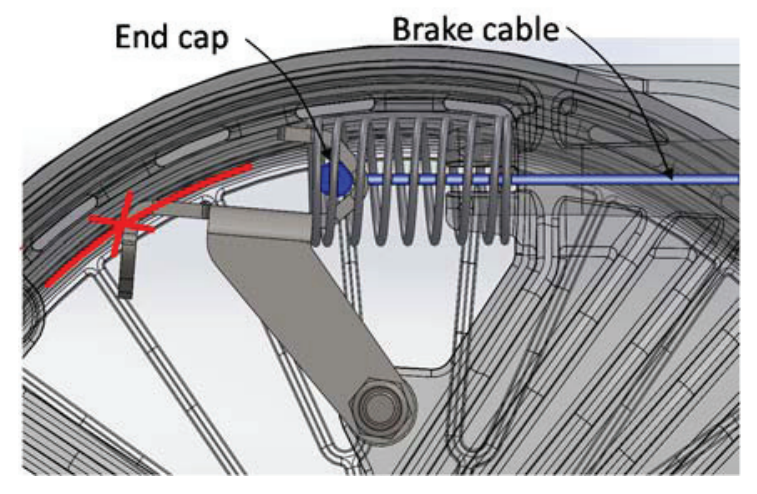

Fig. 8. Brake shoe when engaged.

This extra force on the brake cable ends up forcing the brake shoe to jam into the gap between its pivot point and the inner surface of the wheel rim. The action is equivalent to forcing a self-locking shim between the brake shoe pivot point and the wheel.

Figure 9 shows the nature of the problem. The red circle is drawn concentric to the brake shoe pivot point and to intersect the point of contact between the brake shoe and inner surface of the wheel rim. The tangent directions of the two circles are close, meaning the brake shoe (shim) can easily force its way into the gap between its pivot point (fixed to the frame) and the wheel.

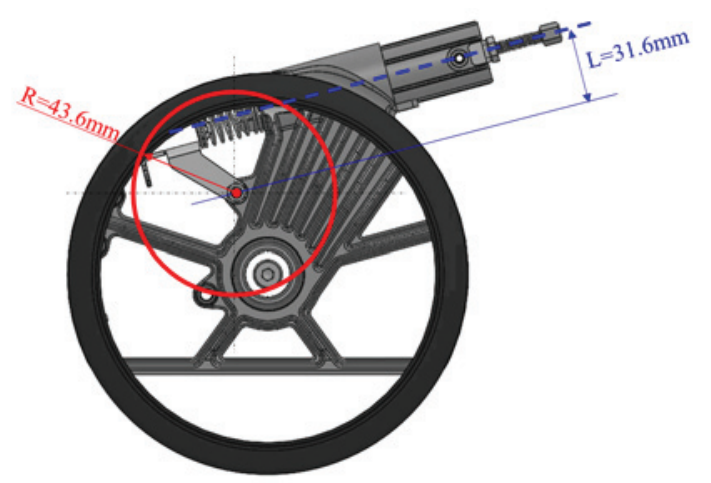

Fig. 9. Original brake shoe pivot point.

As a result, when the user tries to move forward from the rest position, the wheels are locked into position with a shim jammed between the brake shoe pivot point, fixed to the rollator frame, and a wheel. In fact, once the original rollator was stopped by braking, it had to be pulled backwards before making a forward motion. The backwards motion forced the shim out of position and the brake spring then pushed the brake shoe all the way back to its rest position. The spring force of the brake 
spring was not strong enough to brake the shimming force on the brake shoe when it was jammed.

\subsection{Modifying the design}

Axiomatic Design analysis showed us that the problem with the design was an unintended interference of the brake shoe with the FR of moving the rollator forward. It was caused by a poor geometric configuration of the brake shoe, its pivot point, and the point of braking force application.

A modification that quickly comes to the mind is to move the pivot point so that the brake shoe engages the inner surface of the wheel rim at a right angle, instead of an angle close to being tangential to the wheel motion.

In doing so, however, to keep the braking force by the user on the braking lever about the same or smaller, the pivot point has to stay on the lower side of the solid blue line in Figure 9. The lever arm between the line of action of the braking cable and the pivot point has to equal or be larger than the original arm length $\mathrm{L}$ $(31.6 \mathrm{~mm})$ in the figure.

Moving the red circle in Figure 9 around with its center on the solid blue line until the circle intersects with wheel at a right angle gives us the pivot point in Figure 10. Although not impossible to use this configuration, the brake shoe will be considerably larger and it will have to shape itself to work around the fixed frame rib at the 10 o'clock position. This design modification would have violated a geometric constraint in the physical world.

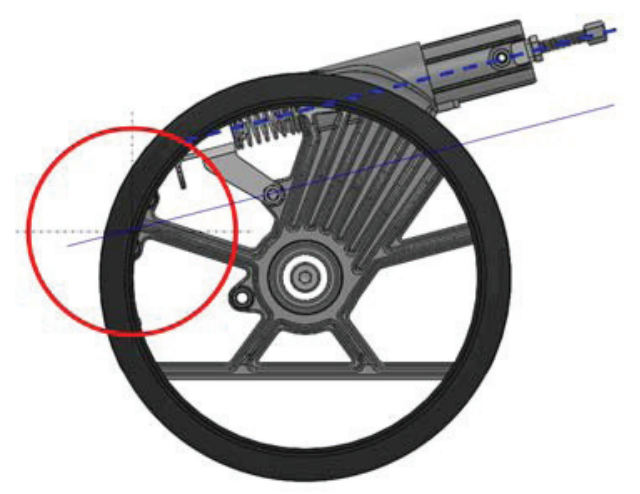

Fig. 10. New brake shoe pivot point candidate.

Figure 11 shows our new design. It has two additional links, and thus, three additional pivot points. In fact, it is a four-bar linkage design that keeps all parts within the area of the original design. Three moving links are clear in the figure, and the fourth is the fixed frame. The original brake shoe pivot point is kept as one of the pivot points on the fixed link. The link that pivots around this pivot point is pulled by the braking cable. The other pivot point on the fixed frame used an already open bolt hole on the frame.

This linkage design kept about the same amount of cable travel necessary to engage the brake and braking force. Figure 12 shows the brake shoe linkage when the brake is engaged. The geometric relation of the new pivot point for the contacting link and point of contact makes the braking force apply normal to the wheel rotation.

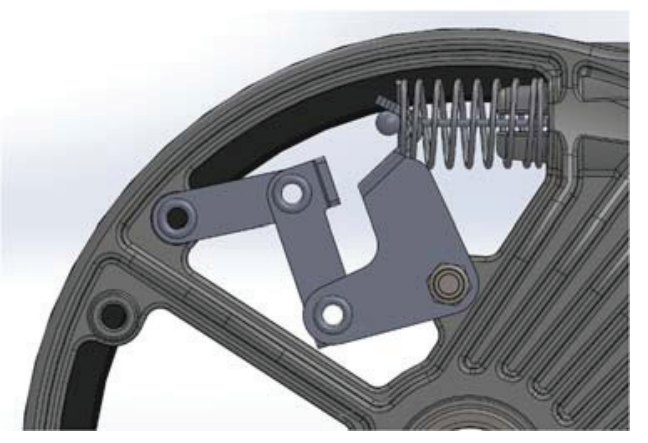

Fig. 11. New four-bar linkage design at rest position.

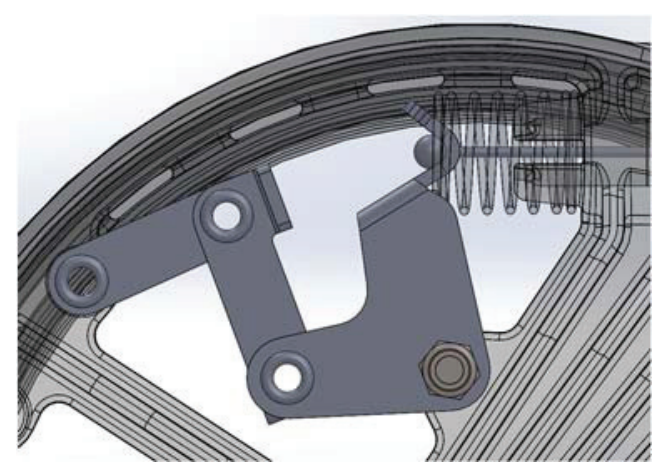

Fig. 12. New four-bar linkage design when brake engaged.

Using an existing wheel, a prototype was built with two dummy wheels and a brake lever to apply braking force to the real wheel. Figure 13 shows the prototype on the left. The right photo in the figure is the details of the three-link brake shoe design when engaged.
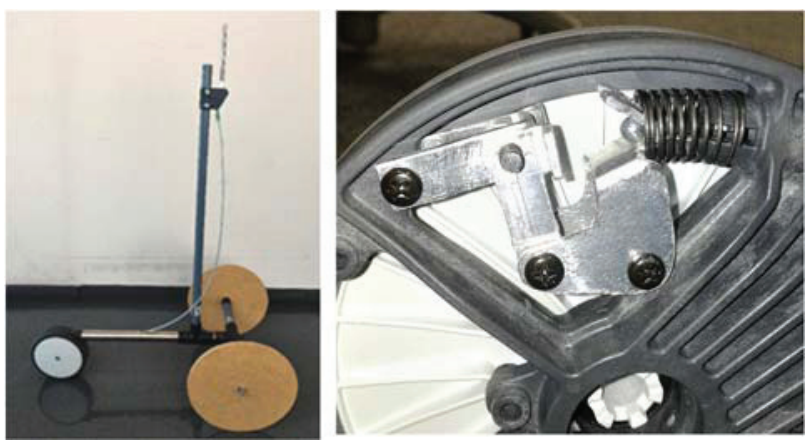

Fig. 13. Prototype (left) and detail of new four-bar linkage brake shoe.

The force the user had to apply to stop the prototype from moving was the same with the new design, when compared to the case with the original braking system mounted on the prototype. The prototype rollator was easy to move forward again from positions stopped by engaging the new brake. Even when the user applied somewhat excessive force to the brake lever, the rollator started to move forward again with ease when the break lever was released. 


\section{Discussion}

\subsection{Axiomatic design analysis of the new design}

Axiomatic Design calls designs with diagonal design equations uncoupled, and those with triangular design equations decoupled. Decoupled designs are also proper except the design parameters (DPs) have to be arranged in a specific order to satisfy FRs [5].

In our case, the original design was decoupled (Eqs. (1) and (3)), however, in our attempt to find the brake shoe to satisfy FRs, the DP was not feasible against a spatial constraint (Figure 10). This attempt was not for satisfying the set of FRs, however, it was aiming to reduce the magnitude of the problematic interference $x$, i.e., the influence of the brake shoe configuration on the FR of making forward movement.

The new four-bar linkage design for the brake shoe also was in efforts of reducing the interfering component $x$ to zero.

The question raised after completing the redesign was if the original design was really proper. If it was decoupled, the product would have satisfied the users but it did not.

What was overlooked in our original analysis was the passive influence of the brake shoe on the function of disengaging the brake shoe from the wheel rim when making a forward movement. This influence comes from the geometric configuration that Figure 9 shows. When the user releases the braking lever, the brake spring failed to disengage the brake shoe. The brake shoe was jammed between the frame and the wheel. This is shown with the red link in Figure 14. The design equation would have been as shown in Eq. (4), i.e., a coupled design.

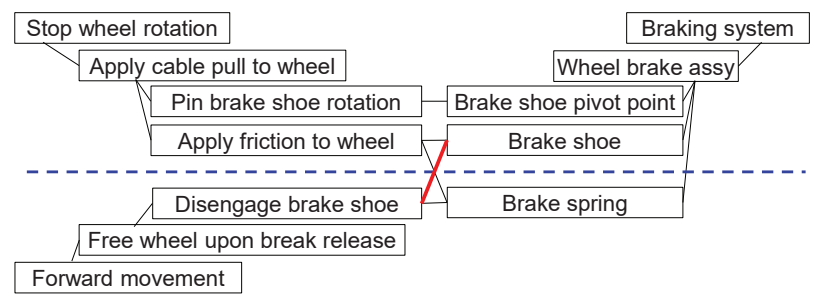

Fig. 14. New four-bar linkage design at rest position.

$\left\{\begin{array}{l}\text { Input user int. to stop } \\ \text { Pin BL rotation } \\ \text { Provide cable path } \\ \text { Transfer pull } \\ \text { Friction to wheel } \\ \text { Pin B shoe rotation } \\ \text { Disengage B shoe }\end{array}\right\}=\left[\begin{array}{lllllll}X & 0 & 0 & 0 & 0 & 0 & 0 \\ 0 & X & 0 & 0 & 0 & 0 & 0 \\ 0 & 0 & X & 0 & 0 & 0 & 0 \\ 0 & 0 & 0 & X & 0 & 0 & 0 \\ 0 & 0 & 0 & 0 & X & 0 & x \\ 0 & 0 & 0 & 0 & 0 & X & 0 \\ 0 & 0 & 0 & 0 & x & 0 & X\end{array}\right]\left\{\begin{array}{l}\text { Brake lever }(\mathrm{BL}) \\ \text { BL pivot point } \\ \text { B sheath } \\ \text { B cable } \\ \text { B shoe } \\ \text { B shoe pivot point } \\ \text { B spring }\end{array}\right\}$

This type of passive interference identified, here in this case, meaning "a DP failing to perform its FR causes a different FR to persist" is difficult to identify.

The redesign with a four-bar linkage brake shoe eliminated the interference and turned the design matrix into a triangular one, back to Eq. (3), i.e., the originally coupled design was made decoupled.

\section{Conclusions}

An existing rollator design was investigated for a graduate student's 3-month project.

The student applied Axiomatic Design in identifying and explaining the problem with the existing design. The rollator, once stopped by engaging its brake, had to be pulled backwards before moving forward again.

The problem was the brake shoe configuration that jammed the wheel rotation upon engagement of the brake.

An attempt of modifying the DP to remove the interference was unacceptable due to spatial constraints.

Redesigning the brake shoe configuration nullified the unintended interference and the design turned into an decoupled design.

The prototype built demonstrated that the new fourbar linkage brake shoe performed equally well in terms of stopping the rollator motion and completely removed the problem of difficulty in restarting the rollator forward from a brake engaged stopped position.

It is difficult to identify passive interference of a DP on FRs. The passive interference we found was a DP failing to meet its FR causing persistence of another FR which would have stopped its function if the DP had succeeded.

\section{References}

1. United Nations, Department of Economic and Social Affairs, Population Division, World Population Prospects: The 2015 Revision, Population by Age Groups - Both Sexes (2015)

2. Cabinet Office, Government of Japan, 2016 Whitepaper: Aged Society (2016)

3. N.P. Suh, Axiomatic Design: Advances and Applications, Oxford University Press (2001)

4. K. Iino and M. Nakao, Design Record Graph and Axiomatic Design for creative design education, Proceedings of ICAD 2016, PROCIR-D-16-00377 (2016)

5. G.S. Shin and G.J. Park, Decoupling process of a coupled design using the TRIZ module, Proceedings of ICAD 2006, ICAD-2006-04 (2006) 